\title{
Synthesis and Hypnotic and Anti-Human Immunodeficiency Virus-1 Activities of $N^{3}$-Substituted 2'-Deoxy-2'-fluorouridines
}

\author{
Yoshiko SAto, ${ }^{a}$ Kunihiko Utsumi,${ }^{a}$ Tokumi Maruyama, ${ }^{*, a}$ Toshiyuki Kimura, ${ }^{b}$ \\ Ikuo YAMAMOTo, ${ }^{b}$ and Douglas D. RichmaN ${ }^{c}$ \\ Department of Pharmaceutical Sciences, Tokushima Bunri University, ${ }^{a}$ Yamashiro-cho, Tokushima 770, Japan, \\ Department of Hygienic Chemistry, Faculty of Pharmaceutical Sciences, Hokuriku University, ${ }^{b}$ Kanazawa 920-11, \\ Japan, and Departments of Pathology and Medicine, University of California, ' San Diego, La Jolla, California \\ 92093-0679, U.S.A. Received September 16, 1993; accepted October 29, 1993
}

Reaction of 9-[3,5-di- $O$-(tetrahydropyran-2-yl)- $\beta$-D-arabinofuranosyl]uracil (2) with diethylaminosulfur trifluoride in the presence of pyridine afforded $2^{\prime}$-deoxy-2'-fluororiboside $3 \mathrm{a}$, from which $2^{\prime}$-deoxy-2'-fluorocytidine (4b) has been synthesized in good yield. Compound 3a was deprotected and subsequently treated with various benzyl halides or 2-chloro-4-fluoroacetophenone to give corresponding $N^{3}$-substituted $2^{\prime}$-deoxy-2'-fluorouridines $5 \mathrm{a}-\mathrm{c}$ and 6. Compounds $5 \mathrm{a}-\mathrm{c}$, as well as 6 , showed weak hypnotic activity in mice. Compound $4 \mathrm{~b}$ showed moderate antiviral activity against human immunodeficiency virus-1 but $3 b, 5 a-c$, and 6 were virtually inactive.

Keywords 2 -deoxy-2'-fluorouridine; hypnotic activity; central nervous system-depressant; anti-human immunodeficiency virus activity

It is of considerable interest to investigate the role of the $2^{\prime}-\mathrm{OH}$ group in ribonucleosides which differentiate RNA from DNA. In this connection, 2'-deoxy-2'-fluoro analogues of nucleosides, oligonucleotides and polynucleotides have been synthesized. ${ }^{12)}$ In studies of the physico-chemical and biological properties, $2^{\prime}$-deoxy-2'fluoronucleosides have been shown to adopt the $3^{\prime}$-endo conformation and the corresponding polynucleotides behave like polyribonucleotides. ${ }^{3)}$ Recently, uridine was identified as a sleep-promoting substances ${ }^{4}$ and some $N^{3}$-substituted uridine, 6-azauridine and thymidine were found to have central nervous system (CNS)-depressant effects. ${ }^{5)}$ But no report concerning $N^{3}$-substituted uridines that contain unusual sugars has appeared. In this paper, we wish to report the synthesis and CNS-depressant effect of $N^{3}$-benzyl-2'-deoxy-2'-fluorouridine $\mathbf{5 a}-\mathbf{c}$ or the $N^{3}$ phenacyl derivative 6 , as well as the anti-HIV-1 activity of 2'-deoxy-2'-fluorouridines $\mathbf{3 b}, 5 \mathbf{a}-\mathbf{c}, \mathbf{6}$ in addition to the cytidine congener $\mathbf{4 b}$.

Chemical Synthesis 2 '-Deoxy-2'-fluorouridine (3b) was prepared by a modification of Hayakawa's method ${ }^{6)}$ using the tetrahydropyran-2-yl group ${ }^{7)}$ for $3^{\prime}$ - and $5^{\prime}$-hydroxyl protection. Thus, $3^{\prime}, 5^{\prime}$-di- $O$-protection of $2,2^{\prime}-O$-cyclouridine (1a) ${ }^{8)}$ with 3,4-dihydro- $2 H$-pyran and subsequent alkaline treatment afforded the arabinoside 2 . Compound 2 was reacted with diethylaminosulfur trifluoride (DAST) in $\mathrm{CH}_{2} \mathrm{Cl}_{2}$ in the presence of pyridine to give an intermediate 3a in $57 \%$ yield. Deprotection of 3a with pyridinium $p$-toluenesulfonate (PPTS ${ }^{9)}$ in EtOH gave $2^{\prime}$ deoxy-2'-fluorouridine $3 \mathbf{b}^{6}{ }^{6,10)}$ By using the procedure of

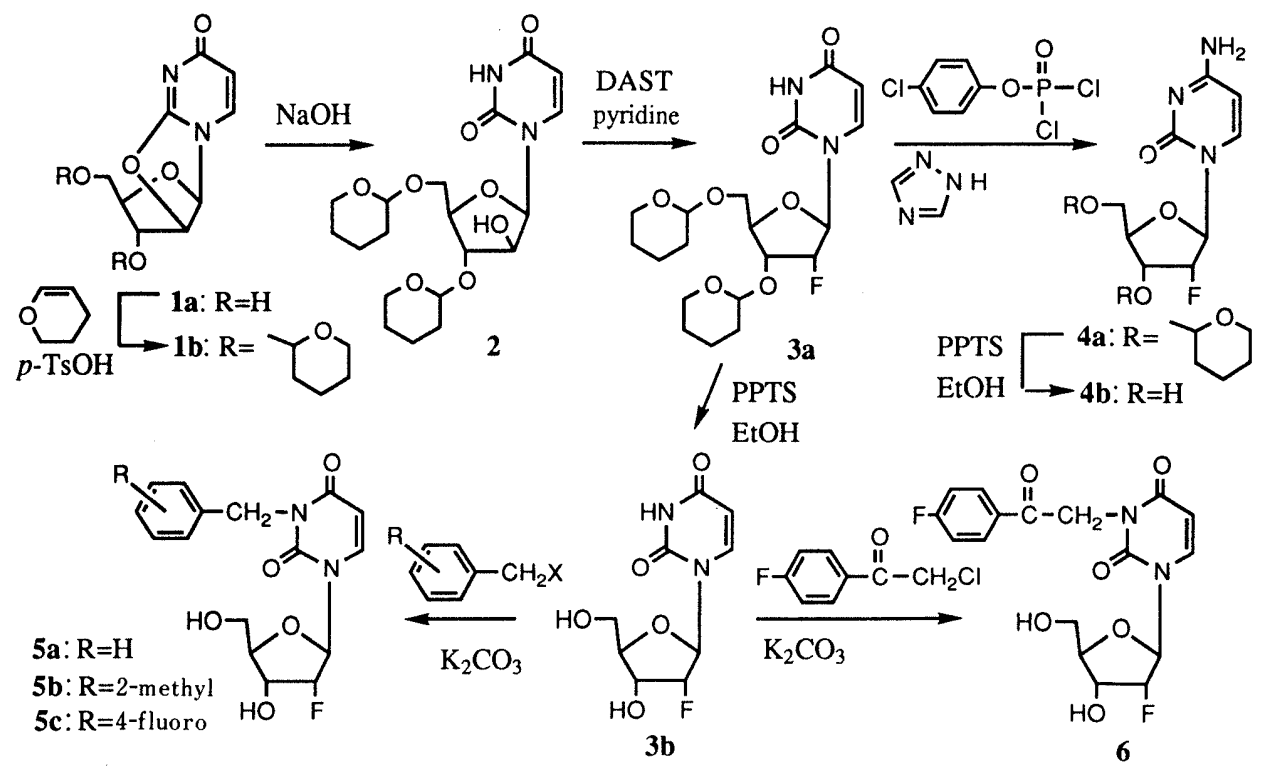

Chart 1

This paper is dedicated to Prof. Leroy B. Townsend on the occasion of his 60 th birthday. 
TABLE I. Reaction Conditions of $N^{3}$-Substituted 2'-Deoxy-2'-fluorouridines

\begin{tabular}{|c|c|c|c|c|c|c|c|}
\hline $\begin{array}{l}\text { Compound } \\
\text { No. }\end{array}$ & $\begin{array}{c}\mathbf{3 b} \\
(\mathrm{mmol})\end{array}$ & $\mathrm{R}-\mathrm{X}$ & $(\mathrm{mmol})$ & $\begin{array}{l}\mathrm{K}_{2} \mathrm{CO}_{3} \\
(\mathrm{mmol})\end{array}$ & Temp. $\left({ }^{\circ} \mathrm{C}\right)$ & Time $(\mathrm{h})$ & Yield $(\%)$ \\
\hline $\mathbf{5 a}$ & 0.5 & $\mathrm{C}_{6} \mathrm{H}_{5} \mathrm{CH}_{2} \mathrm{Br}$ & 0.82 & 0.85 & 50 & 2 & Quantitative \\
\hline $\mathbf{5 b}$ & 0.5 & $o-\mathrm{CH}_{3}-\mathrm{C}_{6} \mathrm{H}_{5} \mathrm{CH}_{2} \mathrm{Cl}$ & 0.75 & 0.85 & 50 & 8 & Quantitative \\
\hline $5 \mathbf{c}$ & 0.5 & $p-\mathrm{F}-\mathrm{C}_{6} \mathrm{H}_{5} \mathrm{CH}_{2} \mathrm{Cl}$ & 0.83 & 0.85 & 50 & 4 & Quantitative \\
\hline 6 & 0.5 & $p-\mathrm{F}-\mathrm{C}_{6} \mathrm{H}_{5} \mathrm{COCH}_{2} \mathrm{Cl}$ & 1.13 & 0.85 & 50 & 4 & 90.4 \\
\hline
\end{tabular}

All reactions were carried out in a mixture of DMF and acetone.

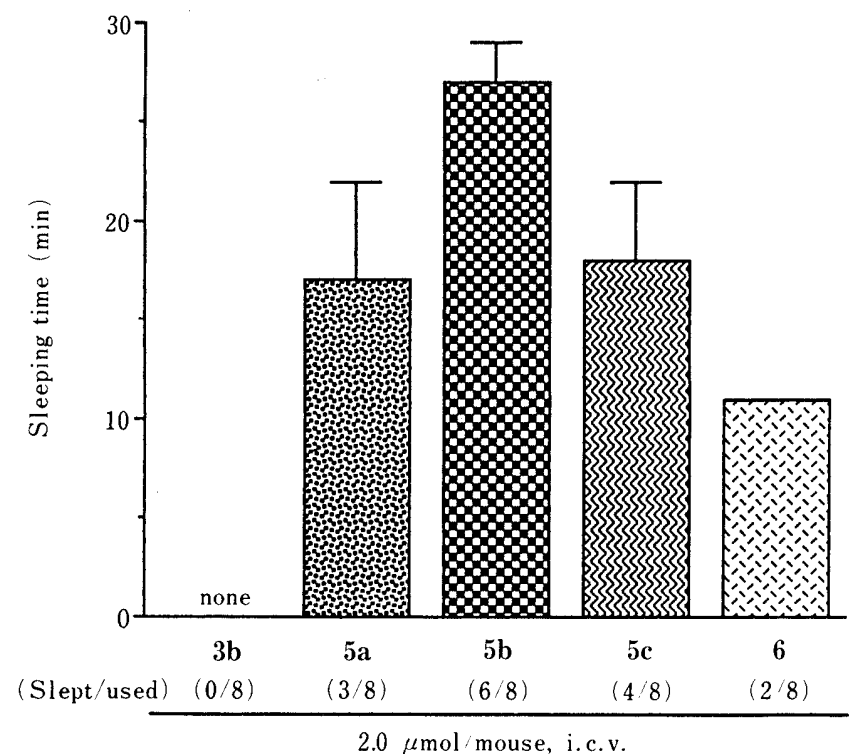

Fig. 1. Hypnotic Activities of $N^{3}$-Substituted 2'-Deoxy-2'-fluorouridines

Mice were administered an $N^{3}$-substituted $2^{\prime}$-deoxy-2'-fluorouridine by i.c.v. injection. The hypnotic activity was expressed as the mean sleeping time of mice $(\min ) \pm$ S.E.

Divakar and Reese, ${ }^{11)}$ 3a was also successively treated with 4-chlorophenyl phosphorodichloridate in the presence of 1,2,4-triazole and concentrated ammonium hydroxide to give the cytidine congener $\mathbf{4 b}{ }^{12)}$ When $\mathbf{3 b}$ was treated with benzyl halides or 2-chloro-4-fluoroacetophenone in the presence of $\mathrm{K}_{2} \mathrm{CO}_{3},{ }^{13)} N^{3}$-substituted $2^{\prime}$-deoxy-2'-fluorouridines $5 \mathbf{a}-\mathbf{c}$ and $\mathbf{6}$ were obtained in good yield.

\section{Pharmacological Results}

Hypnotic Activity of $N^{3}$-Substituted 2'-Deoxy-2'-fluorouridines The hypnotic activity of the $2^{\prime}$-desoxy-2'-fluorouridine and its $N^{3}$-substituted derivatives were assayed according to previously established procedures ${ }^{5)}$ and the results are presented in Fig. 1. In accordance with the result for uridine, ${ }^{5 b)} 2^{\prime}$-deoxy-2'-fluorouridine itself showed no hypnotic activity. But the $N^{3}$-benzyl derivative 5a exhibited hypnotic activity $(17 \mathrm{~min})$ when administered by intracerebroventricular (i.c.v.) injection at $2 \mu \mathrm{M}$ to mice. This is the first demonstration that $N^{3}$-benzyluridine with an unusual sugar has CNSdepressant activity. $N^{3}$-Benzyluridine showed strong hypnotic acitivity $(36 \mathrm{~min})$ in this system. ${ }^{5 b)}$ It appeared that the conversion of the $2^{\prime}-\mathrm{OH}$ to a fluorine atom reduced the CNS-depressant effect by about 50 percent. To explore the effect of substituents on the benzene ring, the hypnotic

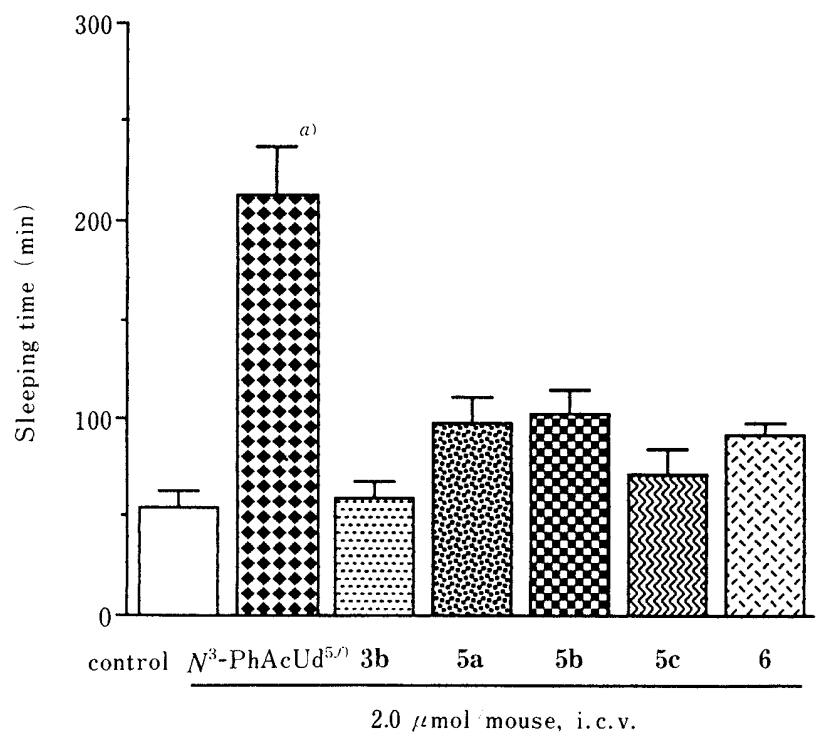

Fig. 2. Effects of $N^{3}$-Substituted $2^{\prime}$-Deoxy-2'-fluorouridine on Pentobarbital-Induced Sleep

Mice were pretreated with $N^{3}$-phenacyluridine ${ }^{5 f)}$ and $N^{3}$-substituted $2^{\prime}$-deoxy2 -fluorouridine $(2.0 \mu \mathrm{mol} / \mathrm{mouse})$ i.c.v. before challenge with sodium pentobarbital $(40 \mathrm{mg} / \mathrm{kg}$, i.p.). Pentobarbital-induced sleep was expressed as the mean sleeping time of mice (min) \pm S.E. a) Significant difference from the control by Bonferroni test, $p<0.01$.

Table II. Plaque Assay Results of Compounds $3 \mathbf{b}, \mathbf{4 b}, \mathbf{5 a}-\mathbf{c}$, and $\mathbf{6}$

\begin{tabular}{cccc}
\hline $\begin{array}{c}\text { Compound } \\
\text { No. }\end{array}$ & $\begin{array}{c}\text { Concentration } \\
(\mu \mathrm{M})\end{array}$ & Average & $\begin{array}{c}\text { Reduction } \\
(\%)\end{array}$ \\
\hline $\mathbf{4 b}$ & 10 & 64 & 43 \\
& 1.0 & 94 & 16 \\
& None & 112 & \\
$\mathbf{3 b}$ & 10 & 109 & 3 \\
$\mathbf{5 a}$ & 10 & 110 & 2 \\
$\mathbf{5 b}$ & 10 & 111 & 1 \\
$\mathbf{5 c}$ & 10 & 124 & 0 \\
$\mathbf{6}$ & 10 & 101 & 10 \\
AZT & 3.16 & 3 & 97 \\
& 1.0 & 4 & 96 \\
& 0.316 & 15 & 87 \\
& 0.1 & 23 & 79 \\
& 0.00316 & 36 & 68 \\
\hline
\end{tabular}

The assay was performed by plaque inhibition assay in CD4 expressing HeLa cells using the HIV-1 $1_{\text {LAI }}$ virus.

activity of $\mathbf{5 b}, \mathbf{c}$ was also examined. The 4-fluorobenzyl derivative $\mathbf{5 c}$ showed equal activity to $\mathbf{5 a}$ but the 2-methylbenzyl derivative $\mathbf{5 b}$ was demonstrated to be more effective than 5a. The $N^{3}$-(4-fluorophenacyl) derivative 6 showed the weakest hypnotic activity among the $N^{3}$-substituted derivatives. 
Effects of $N^{3}$-Substituted $2^{\prime}$-Deoxy-2'-fluorouridine on Pentobarbital-Induced Sleep The effects of the $2^{\prime}$-deoxy$2^{\prime}$-fluorouridine (3b) and its $N^{3}$-substituted derivatives 5a-c are presented in Fig. 2. Here again the $N^{3}$-benzyl derivative showed a short prolongation of pentobarbitalinduced sleep while 2 '-deoxy-2'-fluorouridine itself had no effect. The effect of substituents on the benzene ring was not clear in this sytem.

Anti-Human Immunodeficiency Virus (HIV)-1 Activity The antiviral activity of $\mathbf{3 b}, \mathbf{4} \mathbf{b}, \mathbf{5 a}-\mathbf{c}$, and $\mathbf{6}$ were assayed by HIV plaque reduction in CD4 expressing $\mathrm{HeLa}$ cell monolayers as previously described ${ }^{14)}$ (Tabel II). In this series, compound $\mathbf{4 b}$ displayed indications of activity (43\% reduction) against HIV-1 at $10 \mu \mathrm{M}$. In contrast, the uridine congener $\mathbf{3 b}$ as well as the $N^{3}$-benzyl analogues $5 \mathbf{a}-\mathbf{c}$ and $N^{3}$-phenacyl analog 6 proved inactive against HIV-1.

\section{Experimental}

Melting points $(\mathrm{mp})$ were determined using a Yanagimoto micromelting point apparatus (hot stage type) and are uncorrected. UV spectra were recorded with a Shimadzu UV-190 digital spectrometer Low-resolution mass spectra were obtained on a Shimadzu-LKB 9000B mass spectrometer in the direct-inlet mode. ${ }^{1} \mathrm{H}-\mathrm{NMR}$ spectra were recorded on a Varian UNITY $200(200 \mathrm{MHz})$ or UNITY $600(600 \mathrm{MHz})$ in $\mathrm{CDCl}_{3}$ (or dimethyl sulfoxide (DMSO)- $d_{6}$ ) with tetramethylsilane as an internal standard. Merck Art 5554 plates precoated with Silica gel 60 containing fluorescent indicator $\mathrm{F}_{254}$ were used for thin-layer chromatography and silica gel 60 (Merck 7734, 60-200 mesh) was employed for column chromatography.

$3^{\prime}, 5^{\prime}$-Di- $O$-(tetrahydropyran-2-yl)-2,2'-O-cyclouridine (1b) $p$-Toluenesulfonic acid $(30 \mathrm{~g})$ was added to an ice-cooled solution of $1 \mathrm{a}(37.0 \mathrm{~g}$, $0.16 \mathrm{~mol})$ in $N, N$-dimethylformamide (DMF) $(700 \mathrm{ml})$ and 3,4-dihydro$2 \mathrm{H}$-pyran $(380 \mathrm{ml})$, and the solution was kept at $0{ }^{\circ} \mathrm{C}$ for $2.5 \mathrm{~h}$. After neutralization with triethylamine $(30 \mathrm{ml})$, the solution was subjected to the usual work-up and silca gel chromatography to give a caramel $(56.3 \mathrm{~g}$, $87 \%)$. UV $\lambda_{\max }^{\mathrm{MeOH}} \mathrm{nm}(\varepsilon): 250(7800), \lambda_{\max }^{0.05} \mathrm{~N} \mathrm{HCl}^{\mathrm{O}} \mathrm{nm}(\varepsilon): 251(7700) ;$ $\lambda_{\max }^{0.05 \mathrm{NHCl}} \mathrm{nm}(\varepsilon): 250(8100)$. MS $\mathrm{m} / z: 394\left(\mathrm{M}^{+}\right)$. Anal. Calcd for $\mathrm{C}_{19} \mathrm{H}_{16} \mathrm{~N}_{2} \mathrm{O}_{7}$ : C, 57.86; H, 6.64; N, 7.10. Found: C, 57.84; H, 6.74; $\mathrm{N}$, 7.00

9-[3,5-Di- $O$-(tetrahydropyran-2-yl)- $\beta$-D-arabinofuranosyl]uracil (2) A solution of $1 \mathrm{~b}(56.0 \mathrm{~g}, 0.14 \mathrm{~mol})$ in methanol $(300 \mathrm{ml})$ was treated with $1 \mathrm{~N} \mathrm{NaOH}(180 \mathrm{ml})$ and the solution was stirred at room temperature for $1.5 \mathrm{~h}$, then neutralized with acetic acid $(12 \mathrm{ml})$. The solution was subjected to the usual work-up to give white crystals $(25.1 \mathrm{~g}), \mathrm{mp}$ $187-191^{\circ} \mathrm{C}$. UV $\lambda_{\max }^{\mathrm{MeOH}} \mathrm{nm}(\varepsilon): 262(11000) ; \lambda_{\max }^{0.05} \mathrm{NHCl} \mathrm{nm}(\varepsilon): 262(11300)$; $\lambda_{\max }^{0.05 \mathrm{~N} \mathrm{NaOH}} \mathrm{nm}(\varepsilon): 260(11600)$. MS $m / z: 328\left(\mathrm{M}^{+}-\mathrm{C}_{5} \mathrm{H}_{8} \mathrm{O}\right)$. Anal. Calcd for $\mathrm{C}_{19} \mathrm{H}_{28} \mathrm{~N}_{2} \mathrm{O}_{8}$ : C, 55.33; H, 6.84; N, 6.79. Found: C, 55.13; H, 6.88; $\mathrm{N}, 6.68$. Evaporation of the mother liquor afforded further product as a caramel $(31.1 \mathrm{~g})$.

9-[2-Deoxy-2-fluoro-3,5-di- $O$-(tetrahydropyran-2-yl)- $\beta$-D-ribofuranosyl] uracil (3a) DAST $(16 \mathrm{ml}, 4 \mathrm{eq})$ was added dropwise to a cooled solution $\left(-60^{\circ} \mathrm{C}\right)$ of $2(30.0 \mathrm{~g}, 73 \mathrm{mmol})$ in a mixture of $\mathrm{CH}_{2} \mathrm{Cl}_{2}(370 \mathrm{ml})$ and pyridine $(25 \mathrm{ml})$ under an $\mathrm{N}_{2}$ atmosphere. After heating at reflux for $3 \mathrm{~h}$, the solution was subjected to the usual work-up and silica gel chromatography to give a caramel $(17.2 \mathrm{~g}, 57 \%)$. UV $\lambda_{\max }^{\mathrm{MeOH}} \mathrm{nm}: 262$; $\lambda_{\max }^{0.05 \mathrm{NHCl}} \mathrm{nm}: 262 ; \lambda_{\max }^{0.05 \times \mathrm{NaOH}} \mathrm{nm}: 262$. MS $m / z: 414\left(\mathrm{M}^{+}\right)$.

2'-Deoxy-2'-fluorouridine (3b) A solution of 3a $(3.81 \mathrm{~g}, 9.2 \mathrm{mmol})$ in EtOH $(100 \mathrm{ml})$ was stirred in the presence of PPTS $(1.5 \mathrm{~g})$ at $50{ }^{\circ} \mathrm{C}$ for $1 \mathrm{~d}$. After concentration to $3 \mathrm{ml}$, the solution was chromatographed over a column of Silica gel G $(4.2 \times 28 \mathrm{~cm})$ with $0-20 \% \mathrm{EtOH}$ in $\mathrm{CHCl}_{3}$ (2.4 l) to give a caramel $(1.48 \mathrm{~g}, 66 \%)$. UV $\lambda_{\max }^{\mathrm{MeOH}} \mathrm{nm}: 260 ; \lambda_{\max }^{0.05 \mathrm{~N} \mathrm{HCl}} \mathrm{nm}$ : $260 ; \lambda_{\max }^{0.05 \mathrm{~N} N a \mathrm{H}} \mathrm{nm}: 259 .{ }^{1} \mathrm{H}-\mathrm{NMR}\left(\mathrm{DMSO}-d_{6}\right) \delta: 11.39(1 \mathrm{H}, \mathrm{brs}$, $\left.\mathrm{N}^{3}-\mathrm{H}\right), 7.88(1 \mathrm{H}, \mathrm{d}, J=7.5 \mathrm{~Hz}, \mathrm{H} 6), 5.87(1 \mathrm{H}, \mathrm{d}, J=17.2 \mathrm{~Hz}, \mathrm{HI}), 5.58$ $\left(2 \mathrm{H}, \mathrm{m}, \mathrm{H} 5,3^{\prime}-\mathrm{OH}\right), 5.2\left(1 \mathrm{H}, \mathrm{br} \mathrm{s}, 5^{\prime}-\mathrm{OH}\right), 5.0(1 \mathrm{H}, \mathrm{d}$-like, $J=54.7 \mathrm{~Hz}$, $\left.\mathrm{H} 2^{\prime}\right), 4.12\left(1 \mathrm{H}, \mathrm{m}, \mathrm{H} 3^{\prime}\right), 3.4-3.9\left(3 \mathrm{H}, \mathrm{m}, \mathrm{H}^{\prime}, \mathrm{H} 5^{\prime}\right)$. MS $m / z: 246\left(\mathrm{M}^{+}\right)$.

9-[2-Deoxy-2-fluoro-3,5-di- $O$-(tetrahydropyran-2-yl)- $\beta$-D-ribofuranosyl]cytosine (4a) 4-Chlorophenyl phosphorodichloridate $(9.2 \mathrm{ml})$ was added dropwise to an ice-cooled solution of $3 \mathbf{a}(5.34 \mathrm{~g}, 12.9 \mathrm{mmol})$ and 1,2,4-triazole $(11 \mathrm{~g})$ in pyridine $(70 \mathrm{ml})$, and the solution was stirred at room temperature for $2 \mathrm{~d}$. Usual work-up of the solution gave the triazolidate as a syrup. The product was dissolved in dioxane $(100 \mathrm{ml})$ and concentrated ammonium hydroxide $(33 \mathrm{ml})$ was added to the solution. After stirring at room temperature for $1 \mathrm{~d}$, the solution was subjected to usual work-up and silica gel chromatography to give 4a as a syrup $(5.02 \mathrm{~g}, 94 \%)$. UV $\lambda_{\max }^{\mathrm{MeOH}} \mathrm{nm}: 271 ; \lambda_{\max }^{0.05 \mathrm{~N} \mathrm{HCl}} \mathrm{nm}: 281$; $\lambda_{\max }^{0.05 \mathrm{NaOH}}$ nm: 270 . MS $m / z: 413\left(\mathrm{M}^{+}\right)$.

2'-Deoxy-2'-fluorocytidine (4b) A solution of $4 \mathrm{a}(5.02 \mathrm{~g}, 12 \mathrm{mmol})$ and PPTS $(2.0 \mathrm{~g}, 8 \mathrm{mmol})$ in EtOH $(150 \mathrm{ml})$ was refluxed for $1 \mathrm{~d}$. After evaporation of the solution, the residue thus obtained was dissolved in water $(200 \mathrm{ml})$ and the aqueous layer was passed through a column of Dowex $1\left(\mathrm{OAc}^{-}\right.$form, $\left.50 \mathrm{ml}\right)$. The combined aqueous eluate and washing $(500 \mathrm{ml})$ was concentrated to a small volume to give white crystals $(1.96 \mathrm{~g}$, $66 \%$ ). UV $\lambda_{\max }^{\mathrm{MeOH}} \mathrm{nm}: 270 ; \lambda_{\max }^{0.05 \mathrm{NCl}} \mathrm{nm}: 281 ; \lambda_{\mathrm{NaOH}}^{0.05 \mathrm{NHCl}} \mathrm{nm}: 270.5 . \mathrm{mp}$ $115-120^{\circ} \mathrm{C}$.

Typical Procedure for the Synthesis of $N^{3}$-Substituted 2'-Deoxy-2'fluorouridines Benzyl bromide $(0.1 \mathrm{ml}, 0.82 \mathrm{mmol})$ was added to a solution of $3 \mathrm{~b}(123 \mathrm{mg}, 0.50 \mathrm{mmol})$ and $\mathrm{K}_{2} \mathrm{CO}_{3}(118 \mathrm{mg}, 0.85 \mathrm{mmol})$ in DMF $(2 \mathrm{ml})$ and acetone $(2 \mathrm{ml})$, and the solution was stirred at $50^{\circ} \mathrm{C}$ for $2 \mathrm{~h}$, then cooled. After evaporation of the solution, the residue was dissolved in a small amount of $\mathrm{CHCl}_{3}$ and chromatographed over a column of Silica gel $\mathrm{G}(2.2 \times 15 \mathrm{~cm})$ with $0-10 \% \mathrm{EtOH}$ in $\mathrm{CHCl}_{3}$ $(400 \mathrm{ml})$ to give $N^{3}$-benzyl-2'-deoxy-2'-fluorouridine (5a) as a caramel (172 mg, quantitative). UV $\lambda_{\max }^{\mathrm{MeOH}} \mathrm{nm}(\varepsilon): 261$ (11200); $\lambda_{\max }^{0.05} \mathrm{~N} \mathrm{HCl} \mathrm{nm}(\varepsilon)$ : 261 (11500); $\lambda_{\max }^{0.05 \mathrm{~N} \mathrm{NaOH}} \mathrm{nm}(\varepsilon): 263$ (11600). ${ }^{1} \mathrm{H}-\mathrm{NMR}\left(\mathrm{CDCl}_{3}\right) \delta: 7.55$ $(1 \mathrm{H}, \mathrm{d}, J=8.6 \mathrm{~Hz}, \mathrm{H} 6), 7.23-7.50\left(5 \mathrm{H}, \mathrm{m}, \mathrm{C}_{6} \mathrm{H}_{5} \mathrm{CH}_{2}-\right), 5.81(1 \mathrm{H}, \mathrm{d}$, $J=8.6 \mathrm{~Hz}, \mathrm{H} 5), 5.75-5.85\left(1 \mathrm{H}, \mathrm{m}, \mathrm{Hl}^{\prime}\right), 5.2(1 \mathrm{H}, \mathrm{ddd}, J=53.9,4.9$, $\left.3.2 \mathrm{~Hz}, \mathrm{H} 2^{\prime}\right), 5.10\left(2 \mathrm{H}, \mathrm{d}, \mathrm{C}_{6} \mathrm{H}_{5} \mathrm{CH}_{2}-\right), 4.52\left(1 \mathrm{H}, \mathrm{m}, \mathrm{H} 3^{\prime}\right), 3.98-4.12$ $\left(2 \mathrm{H}, \mathrm{m}, 3^{\prime}-\mathrm{OH}, 5^{\prime}-\mathrm{OH}\right), 3.85\left(1 \mathrm{H}, \mathrm{m}, \mathrm{H} 4^{\prime}\right), 2.52(1 \mathrm{H}, \mathrm{dd}, J=7.0,3.2 \mathrm{~Hz}$, H5'a), $2.38\left(1 \mathrm{H}, \mathrm{dd}, J=7.0,3.8 \mathrm{~Hz}, \mathrm{H} 5^{\prime} b\right)$. MS $m / z: 336\left(\mathrm{M}^{+}\right)$. Anal. Calcd for $\mathrm{C}_{16} \mathrm{H}_{17} \mathrm{FN}_{2} \mathrm{O}_{5} \cdot 1.5 \mathrm{H}_{2} \mathrm{O}: \mathrm{C}, 52.89 ; \mathrm{H}, 5.55 ; \mathrm{N}, 7.71$. Found: C, 52.98; H, 4.85; N, 7.57. .

$N^{3}$-(2-Methyl)benzyl-2'-deoxy-2'-fluorouridine (5b) UV $\lambda_{\max }^{\mathrm{MeOH}} \mathrm{nm}(\varepsilon)$ : $261(13700) ; \lambda_{\max }^{0.05 \mathrm{~N} \mathrm{HCl}} \mathrm{nm}(\varepsilon): 261$ (14100); $\lambda_{\mathrm{NaOH}}^{0.05}{ }^{0.01} \mathrm{~nm}(\varepsilon): 263(14200)$. ${ }^{1} \mathrm{H}-\mathrm{NMR}\left(\mathrm{CDCl}_{3}\right) \delta: 7.61(1 \mathrm{H}, \mathrm{d}, J=8.1 \mathrm{~Hz}, \mathrm{H} 6), 6.93-7.28(5 \mathrm{H}, \mathrm{m}$, $\left.\mathrm{C}_{6} \underline{\mathrm{H}}_{5} \mathrm{CH}_{2-}\right), 5.84(1 \mathrm{H}, \mathrm{d}, J=8.1 \mathrm{~Hz}, \mathrm{H} 5), 5.82(1 \mathrm{H}, \mathrm{dd}, J=18.2,3.5 \mathrm{~Hz}$, $\left.\mathrm{HI}^{\prime}\right), 5.20\left(1 \mathrm{H}, \mathrm{ddd}, J=53.1,4.8,1.8 \mathrm{~Hz}, \mathrm{H2} 2^{\prime}\right), 5.10(2 \mathrm{H}, \mathrm{d}, J=2.5 \mathrm{~Hz}$, $\left.\mathrm{C}_{6} \mathrm{H}_{5} \mathrm{C}_{2}-\right), 4.5\left(1 \mathrm{H}, \mathrm{m}, \mathrm{H}^{\prime}\right), 3.98-4.12\left(2 \mathrm{H}, \mathrm{m}, 3^{\prime}-\mathrm{OH}, 5^{\prime}-\mathrm{OH}\right), 3.85$ $\left(1 \mathrm{H}, \mathrm{m}, \mathrm{H} 4^{\prime}\right), 2.48\left(1 \mathrm{H}, \mathrm{dd}, J=7.1,3.5 \mathrm{~Hz}, \mathrm{H} 5^{\prime} \mathrm{a}\right), 2.35(1 \mathrm{H}, \mathrm{dd}, J=7.1$, $\left.3.3 \mathrm{~Hz}, \mathrm{H}^{\prime} \mathrm{b}\right), 2.42\left(3 \mathrm{H}, \mathrm{s},-\mathrm{CH}_{3}\right)$. MS $m / z: 350\left(\mathrm{M}^{+}\right)$. Anal. Calcd for $\mathrm{C}_{17} \mathrm{H}_{19} \mathrm{FN}_{2} \mathrm{O}_{5} \cdot 0.5 \mathrm{H}_{2} \mathrm{O}: \mathrm{C}, 56.82 ; \mathrm{H}, 5.61 ; \mathrm{N}, 7.80$. Found: $\mathrm{C}, 56.15 ; \mathrm{H}$, $5.49 ; \mathrm{N}, 7.57$.

$N^{3}$-(4-Fluoro)benzyl-2'-deoxy-2'-fluorouridine (5c) UV $\lambda_{\max }^{\mathrm{MeOH}} \mathrm{nm}(\varepsilon)$ : $260(13700) ; \lambda_{\max }^{0.05 \mathrm{~N} \mathrm{HCl}} \mathrm{nm}(\varepsilon): 261(12600) ; \lambda_{\max }^{0.05 \mathrm{~N} \mathrm{NaOH}} \mathrm{nm}(\varepsilon): 262(13400)$. ${ }^{1} \mathrm{H}-\mathrm{NMR}\left(\mathrm{CDCl}_{3}\right) \delta: 7.55(1 \mathrm{H}, \mathrm{d}, J=6.7 \mathrm{~Hz}, \mathrm{H} 6), 6.90-7.50(5 \mathrm{H}, \mathrm{m}$, $\left.\mathrm{C}_{6} \underline{\mathrm{H}}_{5} \mathrm{CH}_{2}-\right), 5.81(1 \mathrm{H}, \mathrm{d}, J=6.7 \mathrm{~Hz}, \mathrm{H} 5), 5.80(1 \mathrm{H}, \mathrm{dd}, J=3.4$, $\left.19.0 \mathrm{~Hz}, \mathrm{H1} 1^{\prime}\right), 5.20\left(1 \mathrm{H}, \mathrm{ddd}, J=4.9,53.7 \mathrm{~Hz}, \mathrm{H} 2^{\prime}\right), 5.05(2 \mathrm{H}, \mathrm{d}$, $\left.\mathrm{C}_{6} \mathrm{H}_{5} \mathrm{CH}_{2}-\right), 4.53\left(1 \mathrm{H}, \mathrm{m}, \mathrm{H} 3^{\prime}\right), 3.98-4.12\left(2 \mathrm{H}, \mathrm{m}, 3^{\prime}-\mathrm{OH}, 5^{\prime}-\mathrm{OH}\right), 3.85$ $\left(1 \mathrm{H}, \mathrm{m}, \mathrm{H} 4^{\prime}\right), 2.42\left(1 \mathrm{H}, \mathrm{dd}, J=5.5,2.9 \mathrm{~Hz}, \mathrm{HS}^{\prime} \mathrm{a}\right), 2.28(1 \mathrm{H}, \mathrm{dd}, J=5.5$, $3.1 \mathrm{~Hz}, \mathrm{H}^{\prime}$ b). $\mathrm{MS} m / z: 354\left(\mathrm{M}^{+}\right)$. Anal. Calcd for $\mathrm{C}_{16} \mathrm{H}_{16} \mathrm{~F}_{2} \mathrm{~N}_{2} \mathrm{O}_{5} \cdot \mathrm{H}_{2} \mathrm{O}$ : C, $51.61 ; \mathrm{H}, 4.87 ; \mathrm{N}, 7.52$. Found: $\mathrm{C}, 51.91 ; \mathrm{H}, 4.71 ; \mathrm{N}, 7.45$.

$N^{3}$-(4-Fluorophenacyl)-2'-deoxy-2'-fluorouridine (6) UV $\lambda_{\max }^{\mathrm{MeOH}} \mathrm{nm}$ : 248; $\lambda_{\max }^{0.05 \mathrm{~N} \mathrm{HCl}} \mathrm{nm}: 249 ; \lambda_{\max }^{0.05 N \mathrm{NaOH}} \mathrm{nm}: 250 .{ }^{1} \mathrm{H}-\mathrm{NMR}\left(\mathrm{CDCl}_{3}\right) \delta: 7.73$ $(1 \mathrm{H}, \mathrm{d}, J=6.9 \mathrm{~Hz}, \mathrm{H} 6), 7.10-8.08\left(5 \mathrm{H}, \mathrm{m}, \mathrm{C}_{6} \underline{\mathrm{H}}_{5} \mathrm{CH}_{2--}\right), 5.85(1 \mathrm{H}, \mathrm{d}$, $J=6.9 \mathrm{~Hz}, \mathrm{H} 5), 5.82(1 \mathrm{H}, \mathrm{dd}, J=14.9,2.4 \mathrm{~Hz}, \mathrm{H1}), 5.20(1 \mathrm{H}$, ddd, $\left.J=43.2,4.2,2.3 \mathrm{~Hz}, \mathrm{H}^{\prime}\right), 5.33\left(2 \mathrm{H}, \mathrm{s}, \mathrm{C}_{6} \mathrm{H}_{5} \mathrm{CH}_{2}-\right), 4.5\left(1 \mathrm{H}, \mathrm{m}, \mathrm{H}^{\prime}\right)$, $3.98-4.12\left(2 \mathrm{H}, \mathrm{m}, 3^{\prime}-\mathrm{OH}, 5^{\prime}-\mathrm{OH}\right), 3.82\left(1 \mathrm{H}, \mathrm{m}, \mathrm{H}^{\prime}\right), 2.6(1 \mathrm{H}, \mathrm{dd}, J=5.7$, $\left.3.2 \mathrm{~Hz}, \mathrm{H} 5^{\prime} \mathrm{a}\right), 2.48\left(1 \mathrm{H}, \mathrm{dd}, J=5.7,2.8 \mathrm{~Hz}, \mathrm{H}^{\prime} \mathrm{b}\right)$. MS $m / z: 382\left(\mathrm{M}^{+}\right)$. Anal. Calcd for $\mathrm{C}_{17} \mathrm{H}_{16} \mathrm{~F}_{2} \mathrm{~N}_{2} \mathrm{O}_{6} \cdot \mathrm{H}_{2} \mathrm{O}: \mathrm{C}, 51.00 ; \mathrm{H}, 4.53 ; \mathrm{N}, 7.00$. Found: C, $51.21 ; \mathrm{H}, 4.12 ; \mathrm{N}, 6.92$.

Hypnotic Activity The compounds tested were suspended in $1 \%$ Tween 80 -saline solution because of their insolubility in saline. The compounds were given by i.c.v. and this method is able to evaluate the real CNS activity without the influence of hepatic metabolism. The sleeping time produced by compounds tested in mice was measured as the time between the loss and recovery of righting reflex.

Effects of Uridine Derivatives on Pentobarbital-Induced Sleep The compound tested was injected, i.c.v., to mice $15 \mathrm{~min}$ before pentobarbital challenge. Sodium pentobarbital was administered intraperitoneally (i.p.) to mice at a dose of $40 \mathrm{mg} / \mathrm{kg}$. Pentobarbital-induced sleeping time in mice was measured as the time between the loss and recovery of the righting reflex. The sleeping time modified by uridine derivatives was 
compared with that of the vehicle control group.

Acknowledgements Tokumi Maruyama was supported by Grants-inAid for Scientific Research on Priority Areas (No. 04226218 and No. 05210211) from the Ministry of Education, Science and Culture, Japan. This study was partially supported by a Grant-in-Aid for Scientific Research to Toshiyuki Kimura from the Ministry of Education, Science and Culture, Japan. Douglas D. Richman was supported by AI-27670, AI-30457, and AI-29164 from the U.S. National Institutes of Health and by the Research Center for AIDS and HIV Infection of the San Diego Veterans Affairs Medicinal Center.

\section{References and Notes}

1) M. Ikehara, Heterocycles, 21, 75 (1984).

2) A. M. Kawasaki, M. D. Casper, S. M. Freier, E. A. Lesnik, M. C. Zounes, L. L. Cummins, C. Gonzalez, P. D. Cook, J. Med. Chem., 36, 831 (1993) and references cited therein.

3) a) W. Guschlbauer, M. Blandin, J. L. Drocourt, M. N. Thang, Nucleic Acids Res., 4, 1933 (1977); b) N. Kakiuchi, C. Marek, N. Rousseau, M. Leng, E. De Clercq, J. Biol. Chem., 257, 1924(1982).

4) Y. Komoda, M. Ishikawa, H. Nagasaki, M. Iriki, K. Honda, S. Inoue, A. Higashi, K. Uchizono, Biomed. Reds., 4, 223 (1983).

5) a) I. Yamamoto, T. Kimura, Y. Tateoka, K. Watanabe, I. K. Ho, Chem. Pharm. Bull., 33, 4088 (1985); b) I. Yamamoto, T. Kimura, Y. Tateoka, K. Watanabe, I. K. Ho, J. Med. Chem., 30, 2227 (1987); c) M. Koshigami, K. Watanabe, T. Kimura, I. Yamamoto, Chem. Pharm. Bull., 39, 2597 (1991); d) I. Yamamoto, T. Kimura, Y. Tateoka, K. Watanabe, I. K. Ho, Res. Commun. Chem. Pathol.
Pharmacol., 52, 321 (1986); e) T. Kimura, K. Watanabe, Y. Takeoka, S. Kondo, I. K. Ho, I. Yamamoto, Chem. Pharm. Bull., 41, 1180 (1993); $f$ ) I. Yamamoto, J. Kuze, T. Kimura, K. Watanabe, S. Kondo, I. K. Ho, Biol. Pharm. Bull., in press.

6) H. Hayakawa, F. Takai, H. Tanaka, T. Miyasaka, K. Yamaguchi, Chem. Pharm. Bull., 38, 1136 (1990).

7) Anti-HIV activity of $2^{\prime}$-deoxy-2'-fluoropurinenucleosides: $T$. Maruyama, K. Utsumi, Y. Sato, D. D. Richman, Nucleosides \& Nucleotides, in press.

8) A. Hampton, A. W. Nichol, Biochemistry, 5, 2076 (1966).

9) M. Miyasita, A. Yosikosi, A. Grieco, J. Org. Chem., 42, 3772 (1977).

10) Preparations of $\mathbf{3 b}$ have been reported: J. F. Codington, I. L. Doerr, J. J. Fox, J. Org. Chem., 29, 558 (1964).

11 K. J. Divakar, C. B. Reese, J. Chem. Soc., Perkin Trans. 1, 1982, 1172.

12) Preparations of $\mathbf{4} \mathbf{b}$ from $2^{\prime}$-deoxy-2'-fluorouridine have been reported; a) M. Blandin, S. Tran-Dinh, J. C. Catlin, W. Guschlbauer, Biochim. Biophys. Acta, 361, 249 (1974); b) I. L. Doerr, J. J. Fox, J. Org. Chem., 32, 1462 (1967), preparations of 4b from 2,2'-O-cyclocytidine hydrofluoride have been reported; c) D. H. Shannahoff, R. A. Sanchez, ibid., 38, 593 (1973); d) R. Mengel, W. Guschlbauer, Angew. Chem. Int. Ed. Engl., 17, 525 (1978).

13) T. Sasaki, K. Minamoto, H. Suzuki, J. Org. Chem., 38, 598 (1973).

14) B. A. Larder, B. Chesebro, D. D. Richman, Antimicrob. Agents \& Chemother., 34, 436 (1990). 